\title{
Prolonged continuous intravenous infusion of the dipeptide L-alanine- L-glutamine significantly increases plasma glutamine and alanine without elevating brain glutamate in patients with severe traumatic brain injury
}

Mirjam Nägeli, Mario Fasshauer, Jutta Sommerfeld, Angela Fendel, Giovanna Brandi and John F Stover

\begin{abstract}
Introduction: Low plasma glutamine levels are associated with worse clinical outcome. Intravenous glutamine infusion dose- dependently increases plasma glutamine levels, thereby correcting hypoglutaminemia. Glutamine may be transformed to glutamate which might limit its application at a higher dose in patients with severe traumatic brain injury (TBI). To date, the optimal glutamine dose required to normalize plasma glutamine levels without increasing plasma and cerebral glutamate has not yet been defined.
\end{abstract}

Methods: Changes in plasma and cerebral glutamine, alanine, and glutamate as well as indirect signs of metabolic impairment reflected by increased intracranial pressure (ICP), lactate, lactate-to-pyruvate ratio, electroencephalogram (EEG) activity were determined before, during, and after continuous intravenous infusion of $0.75 \mathrm{~g}$ L-alanine-L-glutamine which was given either for 24 hours (group $1, n=6$ ) or 5 days (group $2, n=6$ ) in addition to regular enteral nutrition. Lab values including nitrogen balance, urea and ammonia were determined daily.

Results: Continuous L-alanine-L-glutamine infusion significantly increased plasma and cerebral glutamine as well as alanine levels, being mostly sustained during the 5 day infusion phase (plasma glutamine: from $295 \pm 62$ to $500 \pm$ $145 \mu \mathrm{mol} / \mathrm{l}$; brain glutamine: from $183 \pm 188$ to $549 \pm 120 \mu \mathrm{mol} / \mathrm{l}$; plasma alanine: from $327 \pm 91$ to $622 \pm 182 \mu \mathrm{mol} /$ l; brain alanine: from $48 \pm 55$ to $89 \pm 129 \mu \mathrm{mol} / \mathrm{l} ; \mathrm{p}<0.05$, ANOVA, post hoc Dunn's test).

Plasma glutamate remained unchanged and cerebral glutamate was decreased without any signs of cerebral impairment. Urea and ammonia were significantly increased within normal limits without signs of organ dysfunction (urea: from $2.7 \pm 1.6$ to $5.5 \pm 1.5 \mathrm{mmol} / \mathrm{l}$; ammonia: from $12 \pm 6.3$ to $26 \pm 8.3 \mu \mathrm{mol} / \mathrm{l} ; \mathrm{p}<0.05$, ANOVA, post hoc Dunn's test).

Conclusions: High dose L-alanine-L-glutamine infusion $(0.75 \mathrm{~g} / \mathrm{kg} / \mathrm{d}$ up to 5 days) increased plasma and brain glutamine and alanine levels. This was not associated with elevated glutamate or signs of potential glutamate-mediated cerebral injury. The increased nitrogen load should be considered in patients with renal and hepatic dysfunction.

Trial registration: Clinicaltrials.gov NCT02130674. Registered 5 April 2014

\footnotetext{
* Correspondence: john.stover@access.uzh.ch

Surgical Intensive Care Medicine, University Hospital Zuerich, Raemistrasse

100, Zuerich 8091, Switzerland
}

\section{Biomed Central




\section{Introduction}

Low plasma glutamine is associated with increased mortality and aggravated functional impairment in critically ill patients [1]. Thus, adequate substitution of the conditionally essential amino acid glutamine in critically ill patients is strongly recommended by international societies with a grade-A level of evidence [2,3]. Glutamine essentially supports protein synthesis, mitosis, muscle growth, immune function, formation of the antioxidant glutathione, and prevents apoptosis [4]. In addition, glutamine as a gluconeogenic and lipogenic precursor, increases cellular energetic reserves, stimulates insulin release, and ameliorates glucose metabolism under conditions of insulin resistance in critically ill patients $[4,5]$. Glutamine administration reduces infection-related increased morbidity [5-9], decreases mortality during the intensive care phase $[10,11]$, shortens length of hospitalization $[12,13]$, thereby substantially reducing hospital costs [12-14]. The optimal dose, however, still remains to be determined as concluded in the SIGNET trial [15].

Plasma glutamine levels can be increased by enteral as well as intravenous administration of glutamine [16]. However, metabolic conversion of glutamine increases plasma glutamate levels especially during enteral glutamine administration [16]. In patients suffering from a damaged blood-brain barrier as encountered following severe traumatic brain injury (TBI) an increase in plasma glutamate is feared to induce additional brain damage due to its excitotoxic and edema-aggravating potential [17-19]. As shown by Berg and colleagues [20], intravenous infusion of the dipeptide L-alanine-L-glutamine administering $0.34 \mathrm{~g}$ glutamine $/ \mathrm{kg}$ limited to a 20-hour infusion period increased plasma glutamine levels by $30 \%$ without elevating arterial plasma or cerebral glutamate and without signs of cerebral glutamate uptake in patients with severe head trauma (Glasgow coma scale (GCS) score $\leq 8$ ) [21]. In addition, these results suggest that a glutamine dose exceeding $0.34 \mathrm{~g} / \mathrm{kg}$ body weight might be required to normalize decreased plasma glutamine levels in individual patients. However, before a higher glutamine dose can be considered safe in patients suffering from severe TBI, a glutaminedependent increase in cerebral glutamate and signs of cerebral impairment must be excluded during prolonged infusion of a higher glutamine dose. For this, we prospectively investigated the effects of continuous intravenous infusion of $0.5 \mathrm{~g}$ glutamine $/ \mathrm{kg} / \mathrm{d}$ (corresponding to $0.75 \mathrm{~g} / \mathrm{kg} / \mathrm{d}$ Dipeptiven ${ }^{\circ}=\mathrm{L}$-alanine-L-glutamine; $82 \mathrm{mg} /$ $\mathrm{ml} \mathrm{L}$-alanine, $134.6 \mathrm{mg} / \mathrm{ml} \mathrm{L}$-glutamine; Fresenius Kabi, Switzerland) for 24 hours (study 1 , six patients) and 5 days (study 2, six patients). Infusion-related analysis included changes in arterial, jugular venous, and cerebral glutamine, alanine, and glutamate levels as well as alterations in brain glucose, lactate, and pyruvate. In addition, changes in intracranial pressure (ICP), cerebral perfusion pressure
(CPP), brain tissue oxygenation $\left(\mathrm{ptiO}_{2}\right)$, and electroencephalographic activity assessed by bispectral index electroencephalogram (BIS EEG) technology were continuously recorded in patients with traumatic brain injury (TBI) subjected to pharmacologic coma, using fentanyl and midazolam.

\section{Materials and methods}

In study 1 , a total of six patients were included to investigate the effects of $0.5 \mathrm{~g}$ glutamine $/ \mathrm{kg} / \mathrm{d}$ (Dipeptiven ${ }^{\circ}=$ L-alanine-L-glutamine: $82 \mathrm{mg} / 100 \mathrm{ml} \mathrm{L}$-alanine and $134.6 \mathrm{mg} / 100 \mathrm{ml} \mathrm{L}$-glutamine) continuously infused for 24 hours followed by a 24-hour observation period. In study 2, a total of six patients were included to investigate the effects of $0.5 \mathrm{~g}$ glutamine $/ \mathrm{kg} / \mathrm{d}$ (Dipeptiven ${ }^{\circ}=\mathrm{L}$-alanine-L-glutamine; $82 \mathrm{mg}$ L-alanine, $134.6 \mathrm{mg}$ L-glutamine) continuously infused for 5 days followed by a 48-hour observation period. The study protocol was approved by the local ethics committee (Kantonale Ethikkommission Zürich) and written informed consent was obtained by the relatives of the patients.

\section{Inclusion criteria}

Patients suffering from severe TBI reflected by abnormal neurologic status and pathologic neuroradiologic findings were considered eligible when requiring pharmacologic coma. Patients were only included if the different catheters (arterial and central venous lines, neuromonitoring probes; vide infra) and triluminal jejunal feeding tube were in place and functional for at least 12 hours before infusing L-alanine-L-glutamine.

Patients who were anticipated to die within 48 hours with abdominal injury, mass transfusion, renal or hepatic impairment, requiring barbiturates to treat intracranial hypertension, or receiving parenteral nutrition, and having body weight below $50 \mathrm{~kg}$ or exceeding $100 \mathrm{~kg}$ were not included.

\section{Study protocol (study 1 and study 2)}

Following admission to the trauma surgical ICU, all patients were treated according to a standardized protocol as published previously [22,23]. For this, a BIS EEG sensor was applied to guide analgesia and sedation (Figure 1); a jugular venous catheter was inserted within the first hour to guide ventilation and adjust analgesia/sedation and CPP; after approximately 24 hours a control cranial computed tomography (CT) scan was performed to determine the site of insertion of the microdialysis (CMA 70, 10 mm membrane, CMA/ Microdialysis, Dallvägen 10, Solna Sweden) and $\mathrm{ptiO}_{2}$ catheters (LICOX ${ }^{\circ}$ IMC Oxygen Catheter Micro Probe, Integra NeuroSciences, Plainsboro, NJ, USA) to avoid penetrating frontal contusions, as these would not yet be visible on the first CT and tend to develop during the first 24 hours. Microdialysis, $\mathrm{ptiO}_{2}$ and 


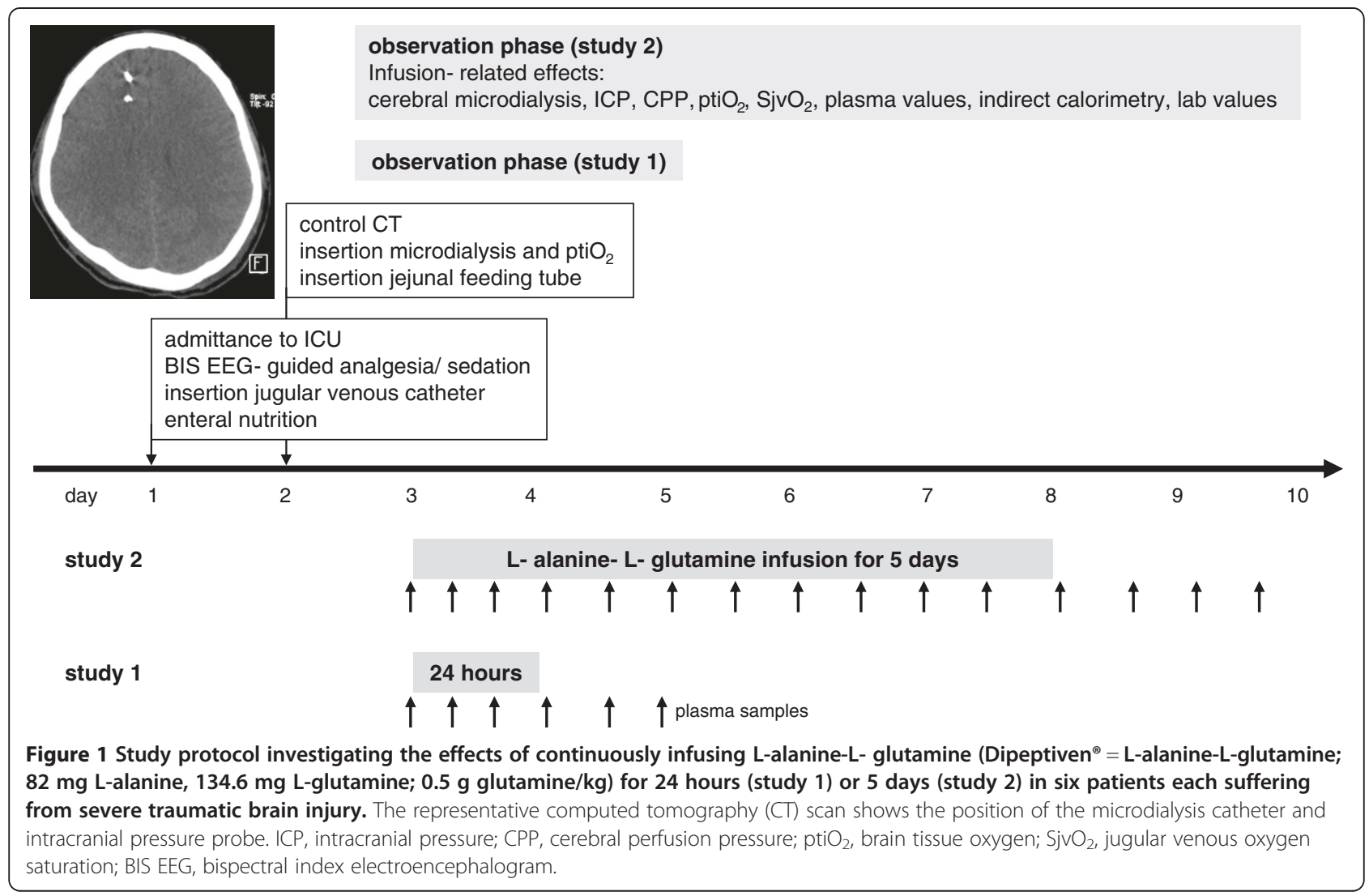

temperature (LICOX ${ }^{\circledR}$ IMC Temperature Micro Probe; Integra NeuroSciences) probes were inserted using a triluminal bolt system (LICOX ${ }^{\odot}$ IMC Bolt System, Triple Lumen; Integra NeuroSciences). The patients were fed enterally via a gastric feeding tube beginning on the day of admission to the ICU, using Fresubin ${ }^{\circ}$ Energy fibre (Fresenius Kabi AG, Switzerland), containing $5.6 \mathrm{~g}$ protein $/ 100 \mathrm{ml}$ with $0.62 \mathrm{~g}$ glutamine $/ 100 \mathrm{ml}$ and $0.2 \mathrm{~g}$ alanine $/ 100 \mathrm{ml}$ with $1.5 \mathrm{kcal} / \mathrm{ml}$. On the second post-traumatic day a jejunal feeding tube was positioned endoscopically by the gastroenterologists and enteral nutrition with Fresubin ${ }^{\circledR}$ Energy fibre was continued. None of the patients received parenteral nutrition in addition to the enteral nutrition. According to our standardized nutritional protocol, enteral nutrition was begun within 12 hours after admission to the ICU, started at $10 \mathrm{ml} / \mathrm{h}$ and increased by $10 \mathrm{ml} / \mathrm{h}$ in 6-hour intervals until reaching the measured caloric requirement determined by indirect calorimetry (Deltatrac $^{\mathrm{TM}}$ II; Datex Instruments, Helsinki, Finland). On the third post-traumatic day, patients in study 1 received L-alanine-L-glutamine at $0.5 \mathrm{~g}$ glutamine $/ \mathrm{kg} / \mathrm{d}$ by continuous intravenous infusion for 24 hours, followed by a 24-hour observation period. In study 2, aimed at excluding an accumulation effect, L-alanine-L-glutamine was continuously infused intravenously at 0.5 g glutamine $/ \mathrm{kg} / \mathrm{d}$ starting on the third posttraumatic day lasting for five days, followed by an additional 48-hour observation phase.

Cerebral microdialysis samples were measured hourly to monitor metabolic alterations reflected by changes in glucose, lactate, pyruvate, and glutamate. The remaining volume was used to analyze changes in alanine, glutamine, and glutamate by high performance liquid chromatography (HPLC). In study 1 , arterial and jugular venous plasma samples were drawn at predefined time points before, during $(1,4,12$, and 23 hours during the infusion period), and after the infusion period (4, 12, and 23 hours after the infusion). Indirect calorimetry was performed before, during (8.0 and 23.5 hours), and after the infusion period (6.0 and 23.5 hours).

In study 2, plasma arterial and jugular venous samples were drawn at predefined time points before, during (1, 4, 12, 24, 36, 48, 60, 72, 84, 96, 108, and 120 hours), and after the infusion period $(4,12,23$, and 48 hours after the infusion). Indirect calorimetry was performed before, during (12-hour intervals), and after the infusion period (12-hour intervals). The results obtained during these investigations were blinded to the clinicians and, thus, did not influence clinical decision-making.

In both studies, predefined signs of glutamate-mediated cerebral injury were actively sought by the study team (JFS, MF, JS) with the aim of stopping the L-alanine-L- 
glutamine infusion in case of cerebral metabolic impairment reflected by elevated cerebral glutamate, increased lactate and lactate/pyruvate ratio, decreased $\mathrm{ptiO}_{2}$, elevated ICP, increased BIS EEG activity, and escalation in therapeutic interventions, respectively. Here, the following threshold values were defined a priori: a 2-fold increase in plasma or cerebral glutamate associated with a 2-fold increase in cerebral lactate or lactate/pyruvate ratio, or ICP $>20 \mathrm{mmHg}$, or $\mathrm{SjvO}_{2}<60 \%$, or arterial-jugular venous oxygen difference $<-0.2$, or BIS EEG $>40$, or a more aggressive treatment approach.

\section{Investigated parameters}

Bedside analysis of glutamate was performed enzymatically using the bedside ISCUSFlex Microdialysis Analyzer (CMA/Microdialysis, Dalvägen 10, Solna, Sweden) to guide possible termination of L-alanine L-glutamine infusion. In addition, metabolic parameters, that is, glucose, lactate, pyruvate, and calculated lactate/pyruvate ratio were also determined enzymatically. Glutamine, glutamate, and alanine were determined in arterial and jugular venous plasma and cerebral microdialysis by HPLC.

ICP, CPP, BIS EEG were recorded continuously using the LabPilot ${ }^{\mathrm{Tm}}$ software (CMA/Microdialysis, Dalvägen 10, Solna, Sweden). $\mathrm{ptiO}_{2}$ (LICOX ${ }^{\circ}$ IMC Oxygen Catheter Micro Probe) and brain temperature (LICOX IMC Temperature Micro Probe) (Integra NeuroSciences) were recorded continuously using the LabPilot ${ }^{\mathrm{Tm}}$ software (CMA/ Microdialysis, Dalvägen 10, Solna, Sweden). Indirect calorimetry was performed using the Deltatrac ${ }^{\mathrm{Tm}}$ II (Datex Intrsuments, Helsinki, Finland), which also allowed us to determine the respiratory quotient (RQ).

Routine laboratory values were determined once daily assessing changes in lymphocytes, creatinine, urea, and ammonia. Nitrogen balance was calculated by subtracting urinary nitrogen determined in urine collected over 24 hours from the daily nitrogen intake (enteral nutrition plus L-alanine-L-glutamine infusion); possible nitrogen loss via, for example, skin and stool were not considered.

\section{HPLC analysis of amino acids}

Plasma samples were drawn using commercially available pre-heparinized syringes (safe PICO Aspirator, Radiometer, Copenhagen, Radiometer Medical ApS, Åkadevej 21, DK- 2700 Brønshøj, Denmark). Following centrifugation at $4^{\circ} \mathrm{C}$ and $10,000 \mathrm{rpm}$ for 10 minutes (HETTICH Zentrifugen Universal 30 F/ RF, HETTICH AG, 8806 Bäch, Switzerland) samples were deproteinized with acetonitrile (plasma 1:7, microdialysate 1:3). Following centrifugation the supernatant was frozen at $-80^{\circ} \mathrm{C}$ until further analysis.

Following unthawing, samples were vortexed and spiked with the internal standard norvaline (NVA), centrifuged, and applied to a vacuum manifold system (Multi-well Filter Plate Vacuum Manifold and Accessories for 96-well
Filter Plates, Pall Corporation, USA, Port Washington, NY 11050) using a vacuum of $77.5 \mathrm{mmHg}$ for 15 seconds. Thereafter the samples were transferred to a cooled autosampler maintained at $4^{\circ} \mathrm{C}$. Before automated injection, samples were incubated with ortho-phthaldialdehyde (OPA) (1:1) for 1 minute. After injection, a stepped gradient at a flow rate of $1.5 \mathrm{ml} / \mathrm{min}$ was applied (buffer B: 0,0 , $30,50,100,0,0 \%$ at $0,0.8,12,16,16.2$, and 17 minutes) with a total run time of 18 minutes. HPLC was linked to a fluorescence detector (Agilent HPLC 1100 Serie, Agilent Technologies AG, Basel, Switzerland) set at $340 \mathrm{~nm}$ (excitation) and $450 \mathrm{~nm}$ (emission wavelength). Stationary phase was a ZORBAX Eclipse AAA column $(4.6 \times 75 \mathrm{~mm}$, 3.5- $\mu \mathrm{m}$ particle size) (Agilent Technologies AG). The following mobile phases were used: buffer A: $5.5 \mathrm{~g}$ sodium dihydrogen phosphate anhydrous $\left(\mathrm{H}_{2} \mathrm{NaO}_{4} \mathrm{P}\right)+11$ Aqua HPLC (purified, filtered distilled water) and buffer $\mathrm{B}$ : $450 \mathrm{ml}$ acetonitrile $+450 \mathrm{ml}$ methanol $+100 \mathrm{ml}$ Aqua HPLC (sodium dihydrogen phosphate anhydrous, acetonitrile, and methanol were purchased from SigmaAldrich Chemie GmbH, Buchs, Switzerland).

A standard mixture of the amino acids (AA) analyzed as an external standard was also spiked using the same internal standard (NVA) as in the plasma and microdialysis samples. OPA, AA and NVA standards were purchased from Agilent Technologies AG, Basel, Switzerland.

\section{Standardized intensive care treatment following severe traumatic brain injury}

All patients were treated on our ICU according to a standardized protocol as published previously [22,23] with integrated extended neuromonitoring [24]. Routine treatment and decision-making were not influenced by the present investigations. Continuously infused midazolam $\left(\right.$ Dormicum $\left.^{\circ}\right)$ and fentanyl $\left(\right.$ Sintenyl ${ }^{\circ}$ ) were tapered according to ICP and BIS EEG values, maintaining ICP values $<15 \mathrm{mmHg}$ and BIS EEG values between 20 and 40. Volume and norepinephrine were adjusted to maintain CPP values between 60 and $90 \mathrm{~mm} \mathrm{Hg}$. Transcranial color-coded Duplex sonography was performed daily to guide CPP (including volume management) and ventilator settings ( $\mathrm{paCO}_{2}$ levels) according to changes in flow velocity determined in the middle cerebral artery [25]. Ventilation, oxygenation, CPP, and blood transfusions were guided by maintaining $\mathrm{ptiO}_{2}>15 \mathrm{mmHg}$.

\section{Statistical analysis}

Results of the different parameters assessed at the predefined time points in the individual patients were presented as box plots. Cerebral glutamine, alanine, and glutamate values were determined by HPLC in hourly collected samples pooled in 4-hour blocks resulting in six values to calculate a median per day per patient; the 
box plots represent six daily median values for the six patients in study 1 and six patients in study 2 . In study 2 , the daily medians are compiled in one box plot after having excluded significant differences during the prolonged infusion phase. Statistical differences in changes before, during and after L-alanine-L-glutamine infusion and between the study groups were evaluated by oneway analysis of variance followed by Dunn's multiple comparison test. Differences were rated significant at $P<0.05$.

\section{Results}

\section{Demographic data}

The investigated twelve patients in the two separate studies were comparable presenting with mixed lesions, predominantly consisting of contusions combined with hemispheric edema. In study 1 , the two female and four male patients suffered from severe TBI (median Abbreviated Injury Score (AIS) 4, range 3 to 5) with a median age of 30 (range 19 to 45 ) and a median body mass index of $21 \mathrm{~kg} / \mathrm{m}^{2}$ (range 19 to 23) required prolonged analgesia and sedation with extended neuromonitoring for a median of 13 days (range 7 to 34 days). Microdialysis and $\mathrm{ptiO}_{2} /$ temperature probes were inserted in the more severely injured hemisphere with two in the left frontal and four in the right frontal lobe, respectively. Patients survived with a median extended Glasgow Outcome Score (GOS) of 6 (range 5 to 8) at 12 months following posttraumatic neurorehabilitation.

In study 2, the two female and four male patients suffered from severe TBI (median AIS 4, range 3 to 5) with a median age of 28 years (range 17 to 42 ) and a median body mass index of $23 \mathrm{~kg} / \mathrm{m}^{2}$ (range 21 to 26) required prolonged analgesia and sedation with extended neuromonitoring for a median of 14 days (range 9 to 37 days). Neuromonitoring probes were inserted in the more severely injured hemisphere with three in the left frontal and three in the right frontal lobe, respectively. Insertion of probes did not induce any additional tissue damage as revealed by subsequent CT imaging. Patients survived with a median extended Glasgow Outcome Score (GOS) of 6 (range 5 to 8 ) at 12 months following post-traumatic neurorehabilitation.

\section{Amount of infused L-alanine-L-glutamine and enteral nutrition}

For the administration of $0.5 \mathrm{~g}$ glutamine $/ \mathrm{kg} / \mathrm{d}$ a median of $13 \mathrm{ml} \mathrm{L}$-alanine-L-glutamine (Dipeptiven ${ }^{\circ}$ ) was infused per hour during the 24-hour (study 1) and 5-day (study 2) group, respectively (Table 1 ). This resulted in a cumulative median amount of $312 \mathrm{ml}$ per day (range 216 to $360 \mathrm{ml}$ ) (study 1) and $1,560 \mathrm{ml}$ for 5 days (range 1,080 to $1,800 \mathrm{ml}$ ) (study 2).

Enteral nutrition was continuously applied without any interruptions, resulting in an overall median amount of $45 \mathrm{ml}$ per hour before, during, and after the L-alanineL-glutamine infusion phase (studies 1 and 2) (Table 1).

\section{Changes in plasma glutamine, alanine, and glutamate levels}

Continuous infusion of L-alanine-L-glutamine significantly and reversibly increased plasma glutamine levels from $295 \pm 62 \mu \mathrm{mol} / \mathrm{L}$ to $395 \pm 175 \mu \mathrm{mol} / \mathrm{L}$ during the 24 -hour phase and to $500 \pm 145 \mu \mathrm{mol} / \mathrm{L}$ during the 5-day infusion phase, reaching the highest values during the 5-day infusion period (Figure 2).

During the 24-hour and 5-day infusion phase plasma alanine was significantly and reversibly increased from $327 \pm 91 \mu \mathrm{mol} / \mathrm{L}$ reaching highest values during the 5 -day infusion period $(622 \pm 182 \mu \mathrm{mol} / \mathrm{L})$ (Figure 3). Following the 24-hour infusion phase, plasma alanine returned to baseline levels whereas plasma alanine levels remained significantly increased following the 5-day infusion phase (Figure 3). Plasma glutamate levels remained unchanged during the entire study period (before infusion, $62 \pm 18$; during infusion, $54 \pm 22$; after infusion, $57 \pm 19 \mu \mathrm{mol} / \mathrm{L}$ ), irrespective of infusion duration.

Overall, there were no significant differences between arterial and jugular venous alanine, glutamine, and glutamate levels (data not shown).

\section{Changes in cerebral glutamine, alanine, and glutamate levels}

During the 24-hour infusion phase (study 1) cerebral glutamine (before infusion, 529 \pm 99 ; during infusion, $549 \pm 120$; after infusion, $532 \pm 110 \mu \mathrm{mol} / \mathrm{L}$ ), alanine (before infusion, $82 \pm 69$; during infusion, $89 \pm 90$; after infusion, $77 \pm 78 \mu \mathrm{mol} / \mathrm{L}$ ), and glutamate (before infusion, $12 \pm 11$; during infusion, $5 \pm 10$; after infusion, $6 \pm 9 \mu \mathrm{mol} / \mathrm{L}$ ) remained unchanged.

In study 2, prolonged L-alanine-L-glutamine infusion was associated with significantly increased cerebral glutamine after the infusion phase (Figure 4), whereas cerebral alanine was significantly increased during and after the infusion phase (Figure 5). Cerebral glutamate was significantly decreased during and after the infusion phase (Figure 6).

\section{Changes in cerebral glucose, lactate, lactate-to-pyruvate ratio}

Extracellular cerebral glucose, lactate, and lactate-topyruvate ratio were not influenced by the L-alanineL-glutamine infusion, irrespective of infusion duration (Table 1).

\section{Changes in neuromonitoring and sedation}

During the 24-hour (study 1) and 5-day infusion phase (study 2) ICP, CPP, and $\mathrm{ptiO}_{2}$ remained unchanged (Table 1). Sedation was slowly reduced on days 8 and 9 
Table 1 Changes in enteral nutrition, neuromonitoring, caloric requirement, and laboratory values before, during, and after prolonged continuous L-alanine-L-glutamine infusion

\begin{tabular}{|c|c|c|c|}
\hline & Before infusion & During infusion & After infusion \\
\hline \multicolumn{4}{|c|}{ Enteral nutrition and L-alanine-L-glutamine infusion } \\
\hline Enteral nutrition $(\mathrm{ml} / \mathrm{h})$ & $42 \pm 8,30,62$ & $45 \pm 11,25,60$ & $47 \pm 15,30,90$ \\
\hline Calories (kcal/d) & $1512 \pm 288,1,080,2,232$ & $1620 \pm 396,900,2,160$ & $1692 \pm 540,1,080,3,240$ \\
\hline L-alanine-L-glutamine (ml/h) & - & $13 \pm 2,9,15$ & - \\
\hline \multicolumn{4}{|l|}{ Neuromonitoring } \\
\hline ICP $(\mathrm{mmHg})$ & $11 \pm 4,4,18$ & $9 \pm 5,0,40$ & $9 \pm 6,0,25$ \\
\hline $\mathrm{CPP}(\mathrm{mmHg})$ & $78 \pm 6,71,90$ & $79 \pm 7,60,98$ & $80 \pm 8,56,110$ \\
\hline Brain temperature $\left({ }^{\circ} \mathrm{C}\right)$ & $35.1 \pm 0.2,34.8,35.4$ & $35.4 \pm 0.26,34.6,36$ & $35.5 \pm 0.5,34.5,37$ \\
\hline $\mathrm{ptiO}_{2}(\mathrm{mmHg})$ & $23 \pm 11,10,40$ & $29 \pm 9,15,65$ & $27 \pm 8,11,57$ \\
\hline BIS & $33 \pm 8,20,45$ & $35 \pm 12,10,94$ & $42 \pm 18,10,89^{*}$ \\
\hline Brain glucose (mM) & $0.76 \pm 0.99 ; 0.5,3.5$ & $1.1 \pm 1.1 ; 0.6,4.6$ & $0.68 \pm 1.0 ; 0.4,6,5$ \\
\hline Brain lactate (mM) & $2.2 \pm 3.5 ; 0.8,11$ & $3.8 \pm 1.6 ; 0.5,8.5$ & $3.2 \pm 1.7 ; 0.5,9.3$ \\
\hline Brain lactate-to-pyruvate ratio & $14 \pm 25 ; 2.4,88$ & $14 \pm 23 ; 2,136$ & $9 \pm 22 ; 3,300$ \\
\hline \multicolumn{4}{|l|}{ Caloric requirement } \\
\hline Kcal & $1,830 \pm 193 ; 1,440,1,940$ & $1,945 \pm 320 ; 1,480,2,810$ & $2,115 \pm 353 ; 1,830,2,830^{*}$ \\
\hline $\mathrm{RQ}$ & $0.84 \pm 0.07 ; 0.76,0.94$ & $0.84 \pm 0.05 ; 0.76,0.96$ & $0.83 \pm 0.07 ; 0.72,0.9$ \\
\hline \multicolumn{4}{|l|}{ Laboratory values } \\
\hline CRP & $149 \pm 79,1.3,203$ & $109 \pm 78 ; 25,371$ & $76 \pm 69 ; 12,269$ \\
\hline Fibrinogen & $4 \pm 1.7 ; 1.7,7.1$ & $6.2 \pm 1.5 ; 2.3,8.5$ & $6.5 \pm 1.8 ; 2.2,11.6$ \\
\hline Albumin (g/L) & $23 \pm 4 ; 13,29$ & $22 \pm 4 ; 14,31$ & $21 \pm 5 ; 14,33$ \\
\hline Blood urea $(\mathrm{mmol} / \mathrm{L})$ & $2.7 \pm 1.6 ; 1.2,7$ & $5.5 \pm 1.5 ; 1.6,9.7^{*}$ & $5.1 \pm 2.2 ; 2.2,10.5$ \\
\hline Ammonia ( $\mu \mathrm{mol} / \mathrm{L})$ & $12 \pm 6.3 ; 6,25$ & $26 \pm 8.3 ; 10,51^{*}$ & $26 \pm 8.4 ; 4,34^{*}$ \\
\hline Creatinine $(\mu \mathrm{mol} / \mathrm{L})$ & $68 \pm 23 ; 53,126$ & $66 \pm 16 ; 42,122$ & $68 \pm 17 ; 45,99$ \\
\hline $\mathrm{GOT}(\mathrm{U} / \mathrm{ml})$ & $44 \pm 61,15,206$ & $42 \pm 40 ; 13,165$ & $60 \pm 53 ; 13,191$ \\
\hline GPT (U/ml) & $20 \pm 50 ; 11,141$ & $19 \pm 64,10,172$ & $18 \pm 58,12,158$ \\
\hline Urine urea $(\mathrm{mmol} / \mathrm{L})$ & $74 \pm 56 ; 40,203$ & $136 \pm 85 ; 31,398$ & $117 \pm 53 ; 36,215$ \\
\hline Nitrogen balance $(\mathrm{g} / \mathrm{d})$ & $8 \pm 3.6 ; 5.9,10.7$ & $10 \pm 1.6 ; 6.9,11$ & $8.5 \pm 3.3 ; 6,16.5$ \\
\hline Lymphocytes $\left(10^{-3} / \mu l\right)$ & $0.9 \pm 0.4 ; 0.37,1.58$ & $0.9 \pm 0.3 ; 0.38,1.59$ & $1.0 \pm 0.7 ; 0.72,3.17$ \\
\hline \multicolumn{4}{|l|}{ Sedation } \\
\hline Midazolam (mg/h) & $90 \pm 20 ; 60,110$ & $90 \pm 20 ; 60,110$ & $70 \pm 20 ; 40,90^{*}$ \\
\hline
\end{tabular}

Nitrogen balance was calculated based on administered nitrogen from protein and amino acids minus nitrogen in urinary urea without including other potential losses via, for example, skin and stool. Results are shown as median \pm standard deviation; range. ${ }^{*} P<0.001$ analysis of variance and post hoc Dunn's test. ICP, CPP, $\mathrm{BIS}, \mathrm{ptiO}_{2}, \mathrm{RQ}$, respiratory quotient; CRP, C-reactive protein; GOT, aspartate aminotransferase; GPT, alanine aminotransferase.

after TBI, resulting in significantly increased BIS values compared to baseline values (Table 1).

\section{Changes in caloric requirement, respiratory quotient, laboratory values, and calculated nitrogen balance}

Compared to baseline values caloric requirement $(\mathrm{kcal} / \mathrm{d})$ was significantly increased over time, reaching the highest values after the 5 -day infusion phase, that is, on days 8 and 9 after TBI (Table 1). Calculated RQ remained unchanged. Hepatic and renal function, determined by changes in aspartate aminotransferase (GOT), alanine aminotransferase (GPT), and creatinine, respectively, remained normal (Table 1).
Urea and ammonia levels were significantly increased during the 5-day L-alanine-L-glutamine infusion phase, which persisted during the post-infusion observation phase (Table 1), remaining well below predefined pathologic threshold values of $15 \mathrm{mmol} / \mathrm{L}$ (urea) and $50 \mu \mathrm{mol} / \mathrm{L}$ (ammonia), respectively. During the 24-hour infusion phase the trend in increased urea and ammonia did not reach statistical significance (urea (median) before infusion, 2.2; during infusion, 3.3; after infusion, $2.9 \mathrm{mmol} / \mathrm{L}$, and ammonia (median) before infusion, 12.8; during infusion, 21.7; after infusion, $18.2 \mu \mathrm{mol} / \mathrm{L}$ ).

The calculated nitrogen balance was positive at all time points and was not changed during the 24-hour and 

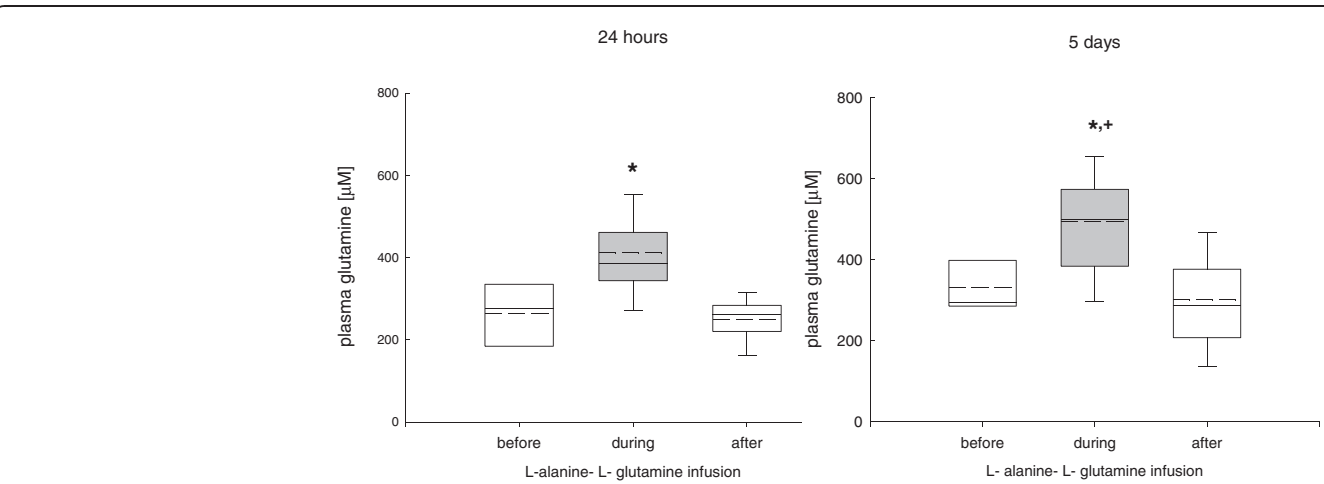

Figure $\mathbf{2}$ Changes in plasma glutamine during the 24-hour and 5-day L-alanine-L-glutamine infusion phase. During the infusion phase plasma glutamine was significantly and reversibly increased compared to baseline values ( ${ }^{*} P<0.001$, analysis of variance, post hoc Dunn's test) which was sustained during the 5 -day compared to the 24-hour infusion phase $\left({ }^{+} P<0.001\right)$.

5-day L-alanine-L-glutamine infusion period (Table 1). The lymphocyte count was not influenced by the 24hour or the 5-day L-alanine-L-glutamine infusion phase, respectively (Table 1 ).

\section{Discussion}

Following severe TBI continuous intravenous infusion of the dipeptide L-alanine-L-glutamine at 0.5 g glutamine/ $\mathrm{kg} / \mathrm{d}$ and $0.25 \mathrm{~g}$ alanine $/ \mathrm{kg} / \mathrm{d}$ significantly and reversibly increased plasma glutamine and alanine concentrations. The increase in plasma glutamine was not associated with elevated plasma or cerebral glutamate or any indirect signs of secondary brain damage reflected by signs of metabolic impairment, increased ICP, decreased $\mathrm{ptiO}_{2}$, or sustained pharmacologic intervention to reduce ICP. Markedly elevated plasma glutamine and alanine were associated with significantly increased cerebral glutamine and alanine levels.

\section{Plasma glutamine and alanine in critically ill patients} Stress and inflammation as well as hormonal changes substantially influence plasma glutamine and alanine pathways to fuel energy-consuming processes [26,27]. Sustained glutamine consumption depletes the muscular glutamine storage and decreases plasma glutamine. Compared to healthy volunteers as published by Stegink et al., [28] and Pouw et al., [29] plasma glutamine was significantly decreased in the critically ill patients suffering from severe TBI, who were investigated in the current study. Hypoglutaminemia as presently shown is in line with the changes seen following, for example, elective surgery [30], neurotrauma [31,32], burn injury [33], sepsis [34], renal insufficiency [35], and chronic pulmonary disease [29]. Hypoglutaminemia, in turn, negatively influences clinical development in critically ill patients, aggravates severity of illness [1], and is associated with increased mortality and morbidity [1], thereby presenting a rationale for corrective strategies.

Contrary to the obvious hypoglutaminemia, baseline plasma alanine determined before L-alanine-L-glutamine infusion was comparable to healthy individuals [28,29] and not reduced following TBI [32]. Normal plasma alanine levels could reflect well-functioning endogenous regulatory processes or successful supply via enteral nutrition,
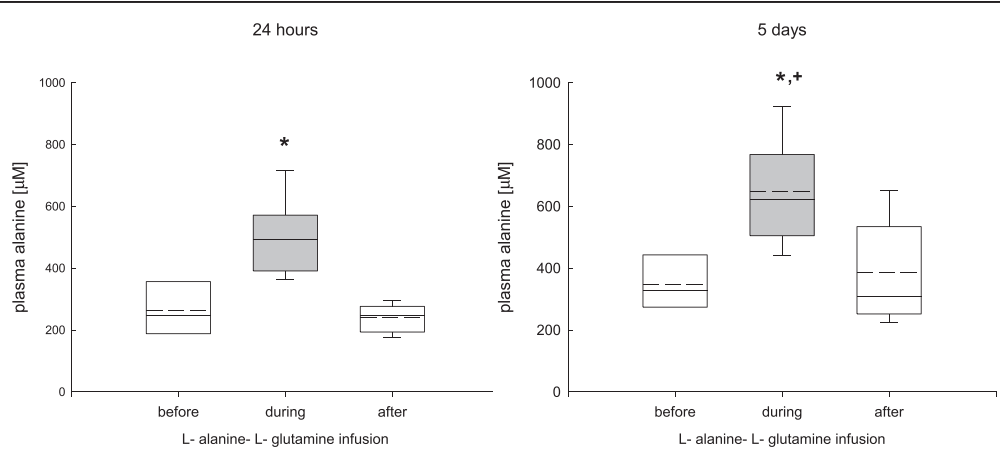

Figure 3 Changes in plasma alanine during the 24-hour and 5-day L- alanine-L-glutamine infusion phase. During the infusion phase plasma alanine was significantly increased compared to baseline values $\left({ }^{*} p<0.001\right.$, analysis of variance, post hoc Dunn's test), which was mostly sustained during the 5-day infusion phase compared to the 24 -hour infusion $\left({ }^{+} P<0.001\right)$. Although elevated plasma alanine levels reached baseline values following the 24-hour infusion phase, plasma alanine levels remained increased following the 5-day infusion phase. 


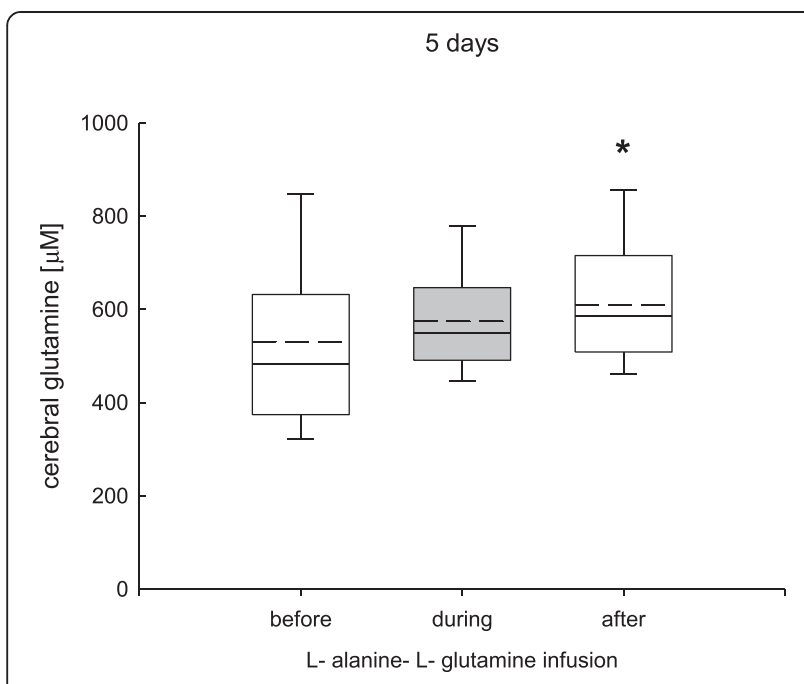

Figure 4 Changes in cerebral glutamine determined by cerebral microdialysis during the 5-day L-alanine-L-glutamine infusion phase. Cerebral glutamine remained unchanged during the infusion phase and showed a significant increase after the 5-day infusion period ( $* P<0.001$, analysis of variance, post hoc Dunn's test).

as all patients had been successfully fed enterally before starting the L-alanine-L-glutamine infusion.

\section{Impact of prolonged L-alanine-L-glutamine infusion on brain parameters}

Brain alanine and glutamine levels were only significantly increased if L-alanine-L-glutamine was infused for 5 days compared to 24 hours. This suggests that prolonged infusion is required to influence brain levels. The persistent increase in brain glutamine and alanine levels could either

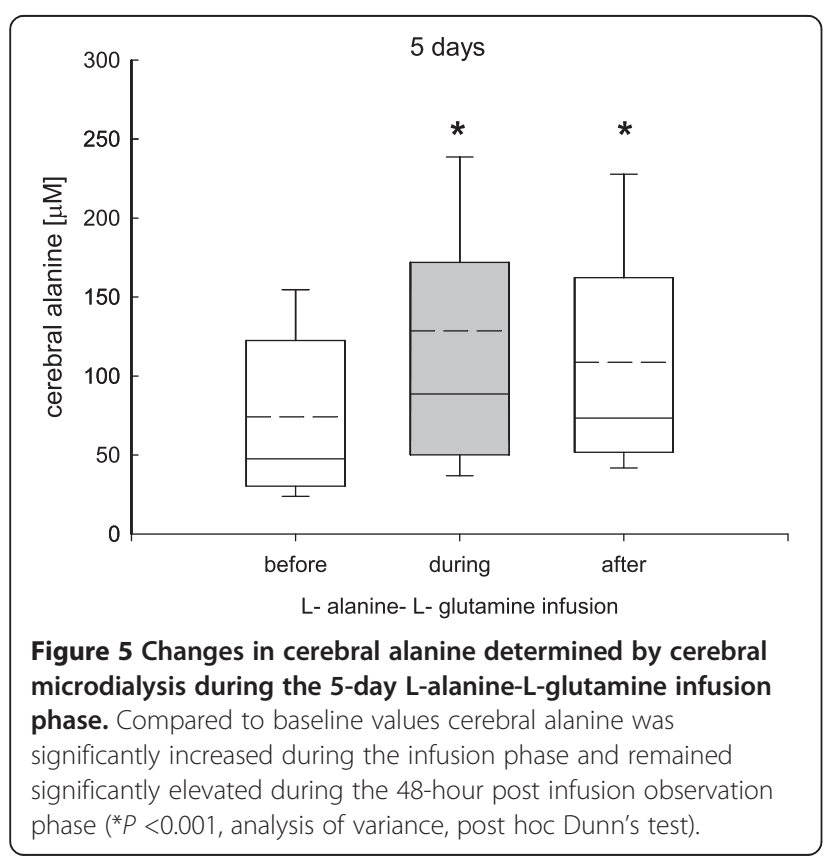

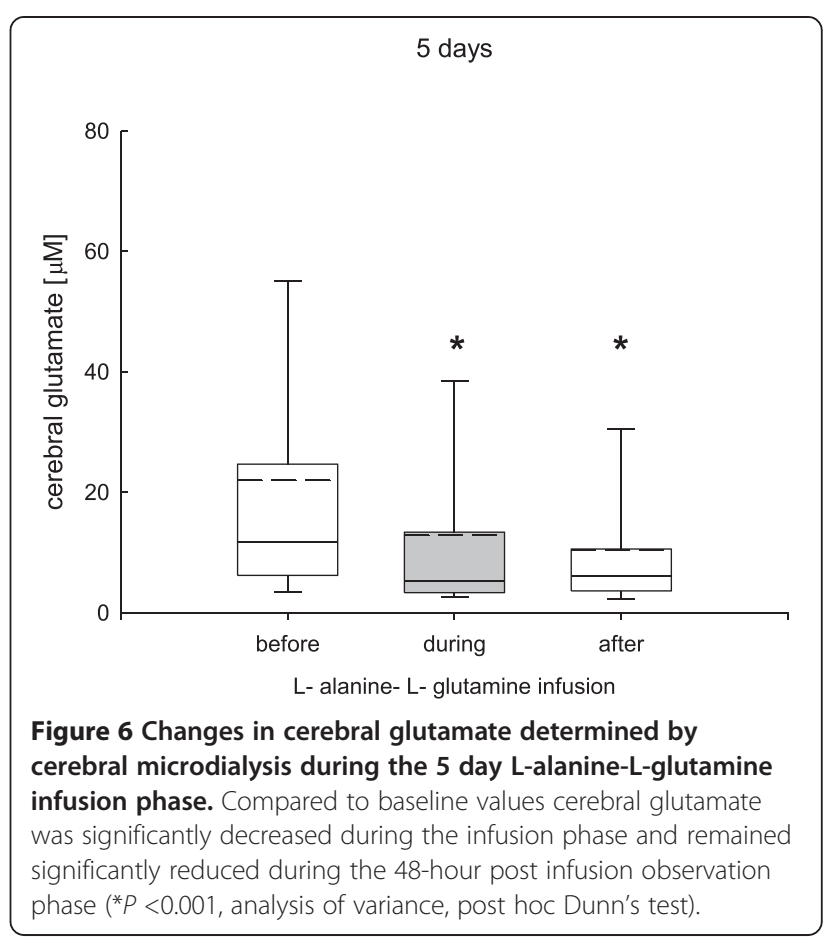

be induced by the infusion itself or by downstream alterations with reduced consumption of glutamine and alanine. Perhaps this pattern reflects time-dependent healing following TBI. However, this cannot be assessed by this study because no control group had been included.

Infusing $0.5 \mathrm{~g}$ glutamine $/ \mathrm{kg} / \mathrm{d}$ for 24 hours and 5 days did not influence measured metabolic parameters and most importantly did not increase brain glutamate levels. In addition, this infusion regimen did not induce cerebral glutamate release as arterio-jugular venous difference remained unchanged. In fact, cerebral glutamate showed a continuous significant decrease over time. Whether this decrease was induced and supported by the present infusion protocol cannot be determined as we did not include a control group. Indirect signs of glutamate-mediated cell injury were absent as reflected by unchanged cerebral glucose, lactate, lactate-to-pyruvate ratio, ICP, CPP, BIS EEG, and absent treatment escalation. This is in line with the results provided by Berg and coworkers in patients with severe head trauma (GCS $\leq 8)[20,21]$. Contrary to the published cerebral glutamine release [21] arterio-jugular venous glutamine differences remained unchanged in the present study. This discrepancy could stem from differences in overall management, including depth of sedation, choice of sedative drugs, cooling, degree of injury, and type of brain lesions. Another possible influence could be the type of nutrition. Whereas Berg and colleagues combined the dipeptide alanine-glutamine with parenteral nutrition, we added alanine-glutamine to enteral nutrition based on the fact that enteral nutrition alone does not 
increase plasma glutamine comparable to its intravenous infusion. Whether the simultaneously infused amino acids in the parenteral nutrition additionally and differentially influences brain metabolism, and specifically glutaminemediated changes, remains to be investigated in a separate study.

The present investigations extend the available knowledge as we investigated a higher dose, that is, $0.5 \mathrm{~g}$ glutamine $/ \mathrm{kg}$ versus $0.34 \mathrm{~g} / \mathrm{kg}$ and a longer duration, that is, 24 hours and 5 days versus 20 hours $[20,21]$. The obtained data show that intravenous infusion of glutamine at a dose higher than currently recommended ( 0.5 versus $0.34 \mathrm{~g} / \mathrm{kg} / \mathrm{d}$ ) does not increase cerebral glutamate or induce glutamatemediated cerebral injury. Thus, this dose and the longer duration appear to be safe in patients with severe TBI.

Infusing a higher dose of alanine, that is, $0.25 \mathrm{~g}$ alanine $/ \mathrm{kg} / \mathrm{d}$ for 24 hours and 5 days compared to $0.17 \mathrm{~g}$ alanine $/ \mathrm{kg} / \mathrm{d}$ for 20 hours [20,21] significantly increased cerebral alanine levels with unchanged arterio-jugular venous difference. Whether the significant and persisting increase in cerebral alanine contributed to the significantly decreased cerebral glutamate by fueling gluconeogenesis, thereby sparing glutamate and glutamine as energydelivering compounds [36,37] or influencing ammonia and lactate transfer [38] cannot be answered by the present study. Increased cerebral alanine levels were not associated with a decrease in BIS EEG values or reduction in administered midazolam dose, which questions its role in neuronal inhibition under the conditions of the present study design [39].

\section{Which glutamine and alanine dose and which duration of infusion could be optimal?}

Contrary to previous investigations, a higher glutamine and alanine dose was infused in the current study, that is, $0.75 \mathrm{~g}$ versus $0.5 \mathrm{~g}$ L-alanine- $\mathrm{L}$-glutamine $/ \mathrm{kg} / \mathrm{d}$ with the aim of normalizing and maintaining normalized plasma glutamine levels. Albeit a significant increase in plasma glutamine even this higher dose, that is, $0.5 \mathrm{~g}$ versus $0.35 \mathrm{~g}$ glutamine $/ \mathrm{kg} / \mathrm{d}$ failed to completely normalize plasma glutamine levels in all patients. This strongly suggests a sustained requirement. Whether this can be met by infusing an even higher glutamine dose in these patients is currently unclear. As shown in non-TBI patients glutamine must be infused at 0.57 or $0.86 \mathrm{~g} / \mathrm{kg} / \mathrm{d}$ to successfully normalize plasma glutamine levels [40].

Plasma alanine was also significantly increased by the currently investigated protocol. Baseline plasma alanine values had already been normal. Thus, the necessity to further increase plasma alanine seems questionable. However, the present study does not allow us to determine potential positive intracellular effects in terms of alaninemediated sustained protein synthesis and decreased protein degradation to define required plasma alanine levels.
Duration of L-glutamine-L-alanine infusion determines persistence of elevated plasma levels. In this context, plasma glutamine returned to baseline values within 30 minutes or 8 hours following a 30 -minute versus a 4-hour infusion, respectively [41,42]. This might explain the significantly increased plasma glutamine and alanine levels during the 5-day versus the 24-hour infusion period observed in the present study. As reflected by the significantly decreased plasma glutamine and alanine levels during the post-infusion period, glutamine and alanine did not accumulate. This is in line with previous reports [43].

Clinical investigations with a larger number of patients are needed to define the optimal dose and duration. The present investigation was not designed to assess the impact of $\mathrm{L}$-alanine-L-glutamine infusion on clinical outcome in this specific population of critically ill TBI patients. The present results could aid in defining the required dose and duration in future clinical trials. The results and the glutamine dose used in the recently published large-scale clinical trials do not provide clear answers as to which glutamine dose is to be used. In the SIGNET trial [15], glutamine is discussed as most likely having been too low. In the REDOXS study, the total glutamine dose from simultaneous enteral and intravenous administration might have been too high in patients suffering from multi-organ failure with unresolved shock, renal failure, and insufficient nutrition [44].

\section{Side effects of prolonged infusion of a higher glutamine and alanine dose}

In line with amino acid-induced ammoniagenesis and ureagenesis [45-48] the presently investigated patients showed increased ammonia and urea levels during Lalanine-L-glutamine infusion compared to baseline values, as they received an average total nitrogen load of $19.7 \mathrm{~g} / \mathrm{d}$ (median enteral nitrogen load $9.7 \mathrm{~g} / \mathrm{d}$, median parenteral nitrogen load from L-alanine-L-glutamine infusion $10 \mathrm{~g} / \mathrm{d}$ ). The elevated urea and ammonia levels did not reach worrisome levels and were not associated with signs of neurologic and metabolic deterioration as ICP remained unchanged, and glutamate was significantly decreased in the face of stable brain metabolism reflected by unchanged cerebral lactate and lactate-to-pyruvate ratio. Apparently, critically ill patients, even with normal hepatic function according to laboratory values and medical history, respond differently to protein load compared to healthy individuals who can tolerate a glutamine load of $0.65 \mathrm{~g}$ glutamine $/ \mathrm{kg} / \mathrm{d}$ without increased plasma ammonia levels [49]. As the increased blood ammonia and urea levels were well within the normal range this metabolic response is not considered a safety concern. The currently investigated patients did not show any signs of hepatic or renal dysfunction. Thus, the investigated dose 
and duration cannot be extrapolated to all patients. The primary aim of these investigations was to determine pharmacodynamic changes. In this context, the missing control group does not allow us to differentiate influences on ammoniagenesis and ureagenesis attributable to nutrition or alanyl-glutamine infusion.

\section{Limitations of the present study}

The low number of patients and the chosen study design without having included a control group does not allow us to recommend the investigated dose and duration in all patients. The presently investigated patients comprise a well-defined subpopulation, which does not allow us to generalize as these patients did not suffer from any additional organ dysfunction apart from brain injury, did not require dialysis or hemofiltration, and had a wellfunctioning gastrointestinal tract receiving enteral nutrition according to the individually determined caloric requirement.

\section{Conclusions}

Low plasma glutamine levels were significantly and reversibly increased by continuous intravenous infusion of $0.75 \mathrm{~g}$ L-alanine-L-glutamine, reaching highest plasma glutamine and alanine levels during the 5-day infusion phase. Infusion of L-alanine-L-glutamine for 5 days was required to significantly increase brain glutamine and alanine levels compared to the 24-hour infusion phase. The absent increase in glutamate as well as absent indirect signs of glutamate-mediated cerebral injury underscore the reported safety of 1) infusing L-alanine-Lglutamine and 2) using the investigated dose in patients with severe TBI. To normalize plasma glutamine levels higher glutamine amounts exceeding $0.5 \mathrm{~g} / \mathrm{kg} / \mathrm{d}$ might be required.

\section{Key messages}

- Continuous intravenous infusion of L-alanine-Lglutamine at $0.75 \mathrm{~g} / \mathrm{kg} / \mathrm{d}$ for 5 days significantly increased plasma as well as cerebral glutamine and alanine levels

- This increase in plasma glutamine was not associated with elevated plasma and cerebral glutamate, and cerebral glutamate release.

- The continuous infusion of L-alanine-L-glutamine at $0.75 / \mathrm{g} / \mathrm{kg} / \mathrm{d}$ for 24 hours significantly increased plasma glutamine and alanine levels without influencing cerebral glutamine and alanine levels.

- Overall, continuous intravenous infusion of a higher L-alanine-L-glutamine dose $(0.75 \mathrm{~g} / \mathrm{kg} / \mathrm{d})$ for up to 5 days was not associated with signs of glutamate-mediated cerebral injury.

\section{Abbreviations}

BIS EEG: bispectral index electroencephalogram; CPP: cerebral perfusion pressure; CT: computed tomography; GCS: Glasgow coma scale; GOT: aspartate aminotransferase; GPT: alanine aminotransferase; HPCL: high performance liquid chromatography; ICP: intracranial pressure; kcal: Kilocalories; $\mathrm{ptiO}_{2}$ : brain tissue oxygen; $\mathrm{RQ}$ : respiratory quotient; $\mathrm{SjvO}_{2}$ : jugular venous oxygen saturation; TBI: traumatic brain injury.

\section{Competing interests}

JFS has received a speaker's honorarium from Fresenius Kabi. Following study completion JFS joined Fresenius Kabi in permanent employment. The remaining authors declare that they have no competing interests.

\section{Authors' contributions}

All authors have contributed substantially to the work: conception (JFS), data acquisition (MN, MF, JS, GB), analysis (AF); all authors contributed to drafting the work and revising it critically for important intellectual content; all authors approved of the final version to be published.

\section{Acknowledgements}

The assistance of the ICU nurses in data collection and Ursula Steckholzer in blood sampling is gratefully acknowledged. This study was supported by the Swiss National Science Foundation (SNF) to JFS, the Division of Surgical Research to JFS, the SUVA Fonds to JFS, and a research grant by Fresenius Kabi Switzerland to JFS. The funding bodies had no role in the design, collection, analysis and interpretation of data, writing of the manuscript, and in the decision to submit the manuscript for publication.

Received: 24 June 2013 Accepted: 2 June 2014

Published: 2 July 2014

\section{References}

1. Rodas PC, Rooyackers O, Hebert C, Norberg A, Wernerman J: Glutamine and glutathione at ICU admission in relation to outcome. Clin Sci (Lond) 2012, 122:591-597. PubMed PMID: 22248298.

2. Kreymann KG, Berger MM, Deutz NE, Hiesmayr M, Jolliet P, Kazandjiev G, Nitenberg G, van den Berghe G, Wernerman J, DGEM (German Society for Nutritional Medicine), Ebner C, Hartl W, Heymann C, Spies C: ESPEN (European Society for Parenteral and Enteral Nutrition). ESPEN Guidelines on Enteral Nutrition: Intensive care. Clin Nutr 2006, 25:210-223.

3. Vanek W, Matarese LE, Robinson M, Sacks GS, Young LS, Kochevar M: Novel Nutrient Task Force, Parenteral Glutamine Workgroup, American Society for Parenteral and Enteral Nutrition (A.S.P.E.N.) Board of Directors. A.S.P.E.N. position paper: parenteral nutrition glutamine supplementation. Nutr Clin Pract 2011, 26:479-494. Epub 2011 Jun 22. PubMed PMID: 21697551.

4. Curi R, Newsholme P, Procopio J, Lagranha C, Gorjão R, Pithon-Curi TC: Glutamine, gene expression, and cell function. Front Biosci 2007, 12:344-357.

5. Déchelotte $P$, Hasselmann M, Cynober $L$, Allaouchiche B, Coëffier $M$, Hecketsweiler B, Merle V, Mazerolles M, Samba D, Guillou YM, Petit J Mansoor O, Colas G, Cohendy R, Barnoud D, Czernichow P, Bleichner G: L-alanyl-L-glutamine dipeptide-supplemented total parenteral nutrition reduces infectious complications and glucose intolerance in critically ill patients: the French controlled, randomized, double-blind, multicenter study. Crit Care Med 2006, 34:598-604.

6. Ziegler TR, Young LS, Benfell K, Scheltinga M, Hortos K, Bye R, Morrow FD, Jacobs DO, Smith RJ, Antin JH, Wilmore DW: Clinical and metabolic efficacy of glutamine-supplemented parenteral nutrition after bone marrow transplantation. A randomized, double-blind, controlled study. Ann Intern Med 1992, 116:821-828.

7. Wischmeyer PE, Lynch J, Liedel J, Wolfson R, Riehm J, Gottlieb L, Kahana M: Glutamine administration reduces Gram-negative bacteremia in severely burned patients: a prospective, randomized, double-blind trial versus isonitrogenous control. Crit Care Med 2001, 29:2075-2080.

8. Fuentes-Orozco C, Anaya-Prado R, González-Ojeda A, Arenas-Márquez H, Cabrera-Pivaral C, Cervantes-Guevara G, Barrera-Zepeda LM: L-alanyl-Lglutamine-supplemented parenteral nutrition improves infectious morbidity in secondary peritonitis. Clin Nutr 2004, 23:13-21.

9. Estívariz CF, Griffith DP, Luo M, Szeszycki EE, Bazargan N, Dave N, Daignault NM, Bergman GF, McNally T, Battey CH, Furr CE, Hao L, Ramsay JG, Accardi 
CR, Cotsonis GA, Jones DP, Galloway JR, Ziegler TR: Efficacy of parenteral nutrition supplemented with glutamine dipeptide to decrease hospital infections in critically ill surgical patients. JPEN J Parenter Enteral Nutr 2008 , 32:389-402.

10. Griffiths RD, Allen KD, Andrews FJ, Jones C: Infection, multiple organ failure, and survival in the intensive care unit: influence of glutaminesupplemented parenteral nutrition on acquired infection. Nutrition 2002, 18:546-552.

11. Griffiths RD, Jones C, Palmer TE: Six-month outcome of critically ill patients given glutamine-supplemented parenteral nutrition. Nutrition 1997, 13:295-302.

12. Goeters C, Wenn A, Mertes N, Wempe C, Van Aken H, Stehle P, Bone HG Parenteral L-alanyl-L-glutamine improves 6-month outcome in critically ill patients. Crit Care Med 2002, 30:2032-2037.

13. MacBurney M, Young LS, Ziegler TR, Wilmore DW: A cost-evaluation of glutamine-supplemented parenteral nutrition in adult bone marrow transplant patients. J Am Diet Assoc 1994, 94:1263-1266.

14. Mertes N, Schulzki C, Goeters C, Winde G, Benzing S, Kuhn KS, Van Aken H, Stehle P, Fürst P: Cost containment through L-alanyl-L-glutamine supplemented total parenteral nutrition after major abdominal surgery: a prospective randomized double-blind controlled study. Clin Nutr 2000 19:395-401.

15. Andrews PJ, Avenell A, Noble DW, Campbell MK, Croal BL, Simpson WG, Vale LD, Battison CG, Jenkinson DJ, Cook JA, Scottish Intensive care Glutamine or seleNium Evaluative Trial Trials Group: Randomised trial of glutamine, selenium, or both, to supplement parenteral nutrition for critically ill patients. BMJ 2011, 342:d1542

16. Melis GC, Boelens PG, van der Sijp JR, Popovici T, De Bandt JP, Cynober L, van Leeuwen PA: The feeding route (enteral or parenteral) affects the plasma response of the dipetide Ala-Gln and the amino acids glutamine, citrulline and arginine, with the administration of Ala-GIn in preoperative patients. Br J Nutr 2005, 94:19-26.

17. Hazell AS: Excitotoxic mechanisms in stroke: an update of concepts and treatment strategies. Neurochem Int 2007, 50:941-953.

18. Kempski O, von Andrian U, Schürer L, Baethmann A: Intravenous glutamate enhances edema formation after a freezing lesion. Adv Neurol 1990, 52:219-223.

19. Stover JF, Sakowitz OW, Kroppenstedt SN, Thomale UW, Kempski OS, Flügge $G$, Unterberg AW: Differential effects of prolonged isoflurane anesthesia on plasma, extracellular, and CSF glutamate, neuronal activity, 125I-Mk801 NMDA receptor binding, and brain edema in traumatic brain-injured rats. Acta Neurochir (Wien) 2004, 146:819-830

20. Berg A, Bellander BM, Wanecek M, Gamrin L, Elving A, Rooyackers O, Ungerstedt $U$, Wernerman J: Intravenous glutamine supplementation to head trauma patients leaves cerebral glutamate concentration unaffected. Intensive Care Med 2006, 32:1741-1746.

21. Berg A, Bellander BM, Wanecek M, Norberg A, Ungerstedt U, Rooyackers $O$, Wernerman J: The pattern of amino acid exchange across the brain is unaffected by intravenous glutamine supplementation in head trauma patients. Clin Nutr 2008, 27:816-821.

22. Meierhans R, Brandi G, Fasshauer M, Sommerfeld J, Schüpbach R, Béchir M, Stover J: Arterial lactate above $2 \mathrm{mM}$ is associated with increased brain lactate and decreased brain glucose in patients with severe traumatic brain injury. Minerva Anestesiol 2012, 78:185-193.

23. Meierhans R, Béchir M, Ludwig S, Sommerfeld J, Brandi G, Haberthür C, Stocker R, Stover JF: Brain metabolism is significantly impaired at blood glucose below $6 \mathrm{mM}$ and brain glucose below $1 \mathrm{mM}$ in patients with severe traumatic brain injury. Crit Care 2010, 14:R13.

24. Stover JF: Actual evidence for neuromonitoring-guided intensive care following severe traumatic brain injury. Swiss Med Wkly 2011, 141:w13245.

25. Brandi G, Béchir M, Sailer S, Haberthür C, Stocker R, Stover JF: Transcranial color-coded duplex sonography allows to assess cerebral perfusion pressure noninvasively following severe traumatic brain injury. Acto Neurochir (Wien) 2010, 152:965-972

26. Bruins MJ, Deutz NE, Soeters PB: Aspects of organ protein, amino acid and glucose metabolism in a porcine model of hypermetabolic sepsis. Clin Sci (Lond) 2003, 104:127-141.

27. Mittendorfer B, Gore DC, Herndon DN, Wolfe RR: Accelerated glutamine synthesis in critically ill patients cannot maintain normal intramuscular free glutamine concentration. JPEN J Parenter Enteral Nutr 1999, 23:243-250.
28. Stegink LD, Filer LJ Jr, Baker GL: Plasma and erythrocyte amino acid levels in normal adult subjects fed a high protein meal with and without added monosodium glutamate. J Nutr 1982, 112:1953-1960.

29. Pouw EM, Schols AM, Deutz NE, Wouters EF: Plasma and muscle amino acid levels in relation to resting energy expenditure and inflammation in stable chronic obstructive pulmonary disease. Am J Respir Crit Care Med 1998, 158:797-801.

30. van Acker BA, Hulsewé KW, Wagenmakers AJ, Soeters PB, von Meyenfeldt MF: Glutamine appearance rate in plasma is not increased after gastrointestinal surgery in humans. J Nutr 2000, 130:1566-1571.

31. Petersen SR, Jeevanandam M, Holaday NJ, Lubhan CL: Arterial-jugular vein free amino acid levels in patients with head injuries: important role of glutamine in cerebral nitrogen metabolism. J Trauma 1996, 41:687-694.

32. Suzuki M, Kudo A, Sugawara A, Yoshida K, Kubo Y, Suzuki T, Ogasawara K, Doi M, Ogawa A: Amino acid concentrations in the blood of the jugular vein and peripheral artery after traumatic brain injury: decreased release of glutamate into the jugular vein in the early phase. J Neurotrauma 2002, 19:285-292.

33. Biolo G, Fleming RY, Maggi SP, Nguyen TT, Herndon DN, Wolfe RR: Inhibition of muscle glutamine formation in hypercatabolic patients. Clin Sci (Lond) 2000, 99:189-194.

34. Druml W, Heinzel G, Kleinberger G: Amino acid kinetics in patients with sepsis. Am J Clin Nutr 2001, 73:908-913.

35. Suliman ME, Qureshi AR, Stenvinkel P, Pecoits-Filho R, Bárány P, Heimbürger O, Anderstam B, Rodríguez Ayala E, Divino Filho JC, Alvestrand A, Lindholm B: Inflammation contributes to low plasma amino acid concentrations in patients with chronic kidney disease. Am J Clin Nutr 2005, 82:342-349.

36. Hertz L: Astrocytic energy metabolism and glutamate formationrelevance for 13C-NMR spectroscopy and importance of cytosolic/mitochondrial trafficking. Magn Reson Imaging 2011, 29:1319-1329.

37. El Hage M, Conjard-Duplany A, Baverel G, Martin G: Metabolic fate of a high concentration of glutamine and glutamate in rat brain slices: a ${ }^{13} \mathrm{C}$ NMR study. Neurochem Int 2011, 58:896-903.

38. Schousboe A, Sonnewald U, Waagepetersen HS: Differential roles of alanine in GABAergic and glutamatergic neurons. Neurochem Int 2003, 43:311-315

39. Tiedje KE, Stevens K, Barnes S, Weaver DF: Beta-alanine as a small molecule neurotransmitter. Neurochem Int 2010, 57:177-188.

40. Tjäder I, Rooyackers O, Forsberg AM, Vesali RF, Garlick PJ, Wernerman J: Effects on skeletal muscle of intravenous glutamine supplementation to ICU patients. Intensive Care Med 2004, 30:266-275.

41. Lochs $\mathrm{H}$, Hübl W: Metabolic basis for selecting glutamine-containing substrates for parenteral nutrition. JPEN J Parenter Enteral Nutr 1990, 14:114S-117S

42. Berg A, Rooyackers O, Norberg A, Wernerman J: Elimination kinetics of L-alanyl-L-glutamine in ICU patients. Amino Acids 2005, 29:221-228

43. Hammarqvist F, Wernerman J, Ali R, von der Decken A, Vinnars E: Addition of glutamine to total parenteral nutrition after elective abdominal surgery spares free glutamine in muscle, counteracts the fall in muscle protein synthesis, and improves nitrogen balance. Ann Surg 1989, 209:455-461. PubMed PMID: 2494960, PubMed Central PMCID: PMC1493967.

44. Heyland D, Muscedere J, Wischmeyer PE, Cook D, Jones G, Albert M, Elke G, Berger MM, Day A: for the Canadian Critical Care Trials Group. A randomized trial of glutamine and antioxidants in critically ill patients. N Engl J Med 2013, 368:1489-1497.

45. Johnston CS, Day CS, Swan PD: Postprandial thermogenesis is increased $100 \%$ on a high-protein, low-fat diet versus a high-carbohydrate, low-fat diet in healthy, young women. J Am Coll Nutr 2002, 21:55-61.

46. Bray GA, Smith SR, de Jonge L, Xie H, Rood J, Martin CK, Most M, Brock C, Mancuso S, Redman LM: Effect of dietary protein content on weight gain, energy expenditure, and body composition during overeating: a randomized controlled trial. JAMA 2012, 307:47-55.

47. Selldén E, Lindahl SG: Postoperative nitrogen excretion after amino acid-induced thermogenesis under anesthesia. Anesth Analg 1998, 87:641-646. 
48. Selldén E, Lindahl SG: Amino acid-induced thermogenesis reduces hypothermia during anesthesia and shortens hospital stay. Anesth Analg 1999, 89:1551-1556.

49. Gleeson M: Dosing and efficacy of glutamine supplementation in human exercise and sport training. J Nutr 2008, 138:2045S-2049S.

doi:10.1186/cc13962

Cite this article as: Nägeli et al:: Prolonged continuous intravenous infusion of the dipeptide L-alanine- L-glutamine significantly increases plasma glutamine and alanine without elevating brain glutamate in patients with severe traumatic brain injury. Critical Care 2014 18:R139.

\section{Submit your next manuscript to BioMed Central and take full advantage of:}

- Convenient online submission

- Thorough peer review

- No space constraints or color figure charges

- Immediate publication on acceptance

- Inclusion in PubMed, CAS, Scopus and Google Scholar

- Research which is freely available for redistribution 